\title{
Quantum Fields with a Multiplicative Structure
}

\author{
W. Rühl and B. C. Yunn
}

Fachbereich Physik, Universität Kaiserslautern, D-6750 Kaiserslautern, Federal Republic of Germany

\begin{abstract}
We define quantum fields (giant fields) on a multidimensional space which contain an infinite set of local fields in Minkowski space. The multiplicative structure for the giant fields implies global expansions for products of the local fields. Conformal symmetry is imposed in order to reduce the number of kinematical variables.
\end{abstract}

\section{Giant Fields and Lie Fields}

We propose to investigate quantum field theories that possess a multiplicative structure

$$
\begin{aligned}
\Phi\left(q_{1}\right) \Phi\left(q_{2}\right)= & H_{1}\left(q_{1}, q_{2}\right) \\
& +\int H_{2}\left(q_{1}, q_{2}, q_{3}\right) \Phi\left(q_{3}\right) d \mu\left(q_{3}\right) .
\end{aligned}
$$

Here $q$ runs over a manifold $Q$ that has more than four dimensions and contains the Minkowski space as a subset. The quantum field $\Phi(q)$ will be called a "giant field". $H_{1}$ and $H_{2}$ are assumed to be corresponding distributions. It is obvious that (1) allows us to express all $n$-point Wightman functions of $\Phi(q)$ in terms of $H_{1}$ and $H_{2}$ provided

$$
\langle 0|\Phi(q)| 0\rangle=0 \text {. }
$$

We assume that this normalization of $\Phi(q)$ is valid and that $\Phi(q)$ is real (symmetric).

The giant field $\Phi(q)$ can in general be decomposed into a bosonic and fermionic part

$$
\Phi(q)=\Phi^{B}(q)+\Phi^{F}(q) .
$$

Equation (1) splits up correspondingly into four equations. In models in which the fermionic part is absent, the antisymmetric parts

$$
\begin{aligned}
H_{1}^{a}\left(q_{1}, q_{2}\right) & =H_{1}\left(q_{1}, q_{2}\right)-H_{1}\left(q_{2}, q_{1}\right) \\
H_{2}^{a}\left(q_{1}, q_{2}, q_{3}\right) & =H_{2}\left(q_{1}, q_{2}, q_{3}\right)-H_{2}\left(q_{2}, q_{1}, q_{3}\right)
\end{aligned}
$$


of $H_{1}$ and $H_{2}$ specify the $n$-point functions completely. In this case (1) implies the Lie structure

$$
\begin{aligned}
{\left[\Phi\left(q_{1}\right), \Phi\left(q_{2}\right)\right]=} & H_{1}^{a}\left(q_{1}, q_{2}\right) \\
& +\int H_{2}^{a}\left(q_{1}, q_{2}, q_{3}\right) \Phi\left(q_{3}\right) d \mu\left(q_{3}\right)
\end{aligned}
$$

Thus a giant field satisfying (1) is in particular a "giant Lie field" [1]. In the case of a mixed bosonic and fermionic giant field the field theory is already fixed once $H_{1}^{B B a}$, $H_{1}^{F F s}, H_{2}^{B B a}, H_{2}^{B F a}, H_{2}^{F F s}$ are given. The kernels $H_{1}$ and $H_{2}$ contain thus much redundant information from the viewpoint of Wightman field theory and must satisfy very restrictive constraints.

In any case this raises the question whether giant fields are beset with the awkward and unwanted properties of standard Lie fields [2,3]. Robinson's theorem [2] applies only to one scalar field and does not apply to giant fields for this trivial reason. Moreover, the variable $q$ involves besides the coordinate $x$ of Minkowski space parameters on which the homogenous Lorentz group acts in a nontrivial fashion. This alone spoils Glaser's arguments [3] which entail that in a standard Lie field model with finitely many field components the angular momentum exchanged in the $S$-channel absorptive part of the four-point Green function is bounded.

In the giant field models that we have in mind primarily and which we propose to study first, we impose an enlarged symmetry on the field, namely conformal covariance. Though we are still sure that none of the arguments brought forward against the standard Lie fields applies to this restricted class of giant fields, we cannot exclude that other yet unknown problematic properties show up.

\section{The Physical Content of Giant Fields}

We imagine a conformally covariant field theory over four dimensional Minkowski space built on $k$ basic fields $\varphi_{i}(x), i=1,2, \ldots, k$. The dynamics of the theory is fixed by covariant global expansions

$$
\begin{aligned}
\varphi_{i}(x) \varphi_{j}(y)= & S_{i j}(x, y) \\
& +\sum_{l=1}^{\infty} \int s_{i j l}(x, y, z) \varphi_{l}^{(i, j)}(z) d^{4} z
\end{aligned}
$$

and by corresponding expansions of products of $\varphi_{l}^{(i, j)}$ with basic fields or with themselves. We shall not discuss here the problems in deriving or proving such expansions [4]. Only few solvable models in two-dimensional Minkowski space have been shown to possess such expansions [5].

Assuming appropriate convergence of the expansions (6) all $n$-point Wightman functions can be expressed in terms of the kernels $s_{i j}, s_{i j l}$. In turn these kernels are determined by covariance requirements up to normalization constants: field normalization constants $\left(s_{i j}\right)$ and coupling constants $\left(s_{i j l}\right)$ [6]. Of course the Wightman axioms are partly taken into account of in the transformation behaviour of the fields. This is fixed (in the infinite sheet formalism [4]) by two invariant parameters, spin and dimension, and eventually internal symmetry quantum numbers. 
Thus in order to define a field theory by expansions (6) one has to specify a countable set of spins and dimensions and give sets of normalization and coupling constants. However, since the axioms have not yet been fully satisfied, these parameters are not all free, but still restricted by "crossing symmetry". Inserting namely (6) into the $n$-point functions in different fashions (which amounts to harmonic analysis of the $n$-point functions on the conformal group in different channels) leads to different expansions that must all be the same.

Deriving from these relations explicit constraints on the spectrum and the normalization constants looks hopeless at present.

We therefore try to reformulate a theory of this kind as a giant field theory. We define the giant field such that it contains the whole set of local fields appearing in the covariant Wilson expansions. To explain how this can be done, we must be a bit more specific $[6,7]$.

We choose as carrier space the homogeneous space

$$
Q=G / B \times Z
$$

of the universal covering group $G$ of the conformal group. $B$ is the subgroup of special conformal transformations, $Z$ is a two-parameter subgroup of homogeneous Lorentz transformations with vanishing eigenvalues for the rotation and boost angles. This homogeneous space possesses an invariant measure $\mu(q)(1)$ and can be parametrized by

$$
q=(n, \lambda, x, \mu, x)
$$

where $n$ is an integer (the sheet label), $\lambda$ is positive real, $x$ and $\mu$ are complex, and $x$ is a Minkowski vector.

Conformal covariance of the giant field is defined by

$$
g \in G \rightarrow \mathscr{U}_{g}: \mathscr{U}_{g} \Phi(q) \mathscr{U}_{g}^{-1}=\Phi\left(q_{g}\right)
$$

where $q \rightarrow q_{g}$ is the action of $G$ on $Q$.

We denote a conformally covariant spinor field of spin $\left(j_{1}, j_{2}\right)$ and dimension $d$ by $\varphi_{n}^{j_{1} j_{2} d}(x)$ in matrix form. Then we postulate the expansion

$$
\begin{gathered}
\Phi_{n}(\lambda, x, \mu, x) \underset{\lambda \rightarrow \infty}{\sim} \sum_{d>\infty} \lambda^{-d-j_{1}-j_{2}} \sum_{\substack{j_{1}, j_{2} \\
\text { finite }}} \\
\times\left[D^{j_{1}}\left(k^{+}\right) \varphi_{n}^{j_{1} j_{2} d}(x) D^{i_{2}}(k)\right]_{j_{1} j_{2}} .
\end{gathered}
$$

Here $D^{j}$ are the usual spinor representation matrices of $\operatorname{GL}(2, C), k$ is a representative element of $\mathrm{GL}(2, C) / Z \times U(1)$, where $U(1)=\{\exp i \arg \operatorname{det} a, a \in \mathrm{GL}(2, C)\}$. $k$ depends on $\varkappa$ and $\mu$.

To give the expansion (10) a meaning we define a conformally invariant test function space [6]. We subject $\mathscr{L}^{2}(Q)$ to harmonic analysis on $G$ and obtain the image space $\mathscr{L}^{2}(\tilde{Q})$,

$$
\tilde{Q}=X \times Q_{6} .
$$

$Q_{6}$ is a homogeneous space of $G$ on which we can define the representations of the continuous principal series in certain $\mathscr{L}^{2}$-spaces. Each point of $X$ denotes one such representation $\chi, \chi$ involves two real continuous parameters $\varrho$ and $\varrho^{\prime}$. Conformal 
invariance necessitates that test functions have a definite asymptotic behaviour in the $Q_{6}$ variables for fixed $\chi$ (see the "canonical" construction of Ref. [6], Appendix). On the other hand we are free to choose the dependence on $X$. We start from $\mathscr{D}_{L^{2-}}$ type functions on $X$ and project out by Hilbert transformations the subspace of analytic functions over $H$ : i.e. analytic functions in $\varrho$ for $\operatorname{Im} \varrho<0$ and in $\varrho^{\prime}$ for $\operatorname{Im} \varrho^{\prime}>0$. Call this space $\mathscr{A}_{H}(\tilde{Q})$. The inverse image of $\mathscr{A}_{H}(\tilde{Q})$ in $\mathscr{L}^{2}(Q)$ is $\mathscr{A}_{H}(Q)$.

Since the Minkowski vectors are contained in $Q_{6}$ and the analytic dependence occurs solely in invariant parameters, we can formulate a Wightman giant field theory with the space $\mathscr{A}_{H}(Q)$ without modification of the other axioms. In order to build in the algebraic structure (1) we require that the kernel $H_{1}$ maps $\mathscr{A}_{H}(Q)$ into $\mathscr{A}_{H}^{\prime}(Q)$ and that $H_{2}$ maps $\mathscr{A}_{H}(Q) \times \mathscr{A}_{H}(Q)$ into $\mathscr{A}_{H}(Q)$ continuously. This can be arranged (Section 3).

Consider an irreducible Wightman representation of the giant field algebra over $\mathscr{A}_{H}(Q)$ in the Hilbert space $\mathfrak{H}$ with the invariant cyclic vector $|0\rangle$. With additional technical assumptions on $H_{1}$ and $H_{2}$ (meromorphy in $\chi$, Section 3 ) we can derive (10). Each term on the r.h.s. arises from poles in $\varrho$ and $\varrho^{\prime}$ in $H$ and transforms as a discrete series representation.

We close this section with a remark on locality. For the space (7) and in view of the expansion (10) we define bosonic and fermionic parts of a giant field by

$$
\begin{aligned}
& \Phi^{B}(q)=\frac{1}{2}\left[\Phi_{n}(\lambda, x, \mu, x)+\Phi_{n}(\lambda,-x,-\mu, x)\right] \\
& \Phi^{F}(q)=\frac{1}{2}\left[\Phi_{n}(\lambda, x, \mu, x)-\Phi_{n}(\lambda,-\chi,-\mu, x)\right] .
\end{aligned}
$$

The locality condition applies to fields with equal $n$ only. The fields on the r.h.s. of (10) with a fixed $n$ belong to the same Borchers class.

\section{Constraints for $\mathrm{H}_{1}$ and $\mathrm{H}_{2}$}

We assume that we have an irreducible representation of the giant field algebra as considered in Section 2. The associative law applied to (1) yields

and

$$
\begin{aligned}
& \int H_{1}\left(q_{1}, q\right) H_{2}\left(q_{2}, q_{3}, q\right) d \mu(q) \\
& \quad=\int H_{2}\left(q_{1}, q_{2}, q\right) H_{1}\left(q, q_{3}\right) d \mu(q)
\end{aligned}
$$

$$
\begin{aligned}
& \int H_{2}\left(q_{1}, q_{2}, q\right) H_{2}\left(q, q_{3}, q_{4}\right) d \mu(q)+H_{1}\left(q_{1}, q_{2}\right) \delta\left(q_{3}, q_{4}\right) \\
& =\int H_{2}\left(q_{2}, q_{3}, q\right) H_{2}\left(q_{1}, q, q_{4}\right) d \mu(q)+H_{1}\left(q_{2}, q_{3}\right) \delta\left(q_{1}, q_{4}\right) .
\end{aligned}
$$

Harmonic analysis on $G$ is applied to both $H_{1}$ and $H_{2}$. From covariance and the spectral condition we obtain a spectral representation of $H_{1}$ in terms of one type of intertwining operator and a weight function $\gamma(\chi)$. The representation of the giant field algebra on $\mathfrak{H}$ requires $H_{1}$ to generate a positive sesquilinear form on $\mathscr{A}_{H}(Q)$ which can be fulfilled by a meromorphic function $\gamma(\chi)$ over $H$ with first order poles and positive residues.

Correspondingly $\mathrm{H}_{2}$ possesses a spectral representation in terms of trilinear covariant kernels. For the purpose of analytically continuing this spectral representation in $\chi_{3}$ into $H$ we have to use covariant kernels of the second kind [8]. Invariance of the space $\mathfrak{H}$ under the multiplication (1) can be achieved by an 
appropriate meromorphic ansatz for the spectral functions $\eta\left(\chi_{1}, \chi_{2}, \chi_{3}\right)^{1}$. Neither positivity nor the spectrum condition constrains $H_{2}$ provided (12) and (13) are fulfilled. Locality can be taken into account by proper choice of the covariant kernels.

By harmonic analysis the condition (12) reduces to an algebraic equation for $\gamma$ and $\eta$. Since this is to be understood in a distribution sense only, it reduces to algebraic relations for the residues of $\gamma$ and $\eta$ in the case that these are realized by meromorphic functions. Condition (13) expresses "crossing symmetry" (or "duality"). It reduces to an integral equation involving pairs of trilinear covariant kernels of the second kind folded together in two different fashions. The relation among two differently folded pairs of covariant trilinear kernels of the first kind can be dealt with for the conformal group in two-dimensional Minkowski space by analytically continued $6 j$-symbols of the rotation group. Nothing explicit is known for higher dimensional spaces nor for any space if second kind kernels are used. Thus we are not able to solve (13) at present for technical reasons. A model study in two-dimensional Minkowski space is of no great interest whatever the result of such investigation is : since it is known that there exist non-pathological models of Lie field theories in two-dimensional Minkowski space [3] (in the case of existence of a solution) and because of the high degeneracy of the spin-dimension spectrum that may not fit into an expansion (10) (in the case there is no solution to our conditions).

We emphasize that meromorphy of the spectral functions of $H_{1}$ and $H_{2}$ with first order poles on the imaginary axis [7] is an additional requirement. It allows us to deduce (6) and (10) instead of Laplace integral representations or even more complicated expressions.

\section{References}

1. Greenberg,O.W.: Ann. Phys. 16, 158 (1961)

2. Robinson,D.W.: Phys. Lett. 9, 189 (1964)

3. Wightman, A.S. : Introduction to some aspects of the relativistic dynamics of quantized fields. In: Cargèse lectures in theoretical physics, high energy electromagnetic interactions and field theory (ed. M. Lévy). New York: Gordon and Breach 1967

4. In general the expansions (6) involve non-local projections of local fields or equivalently local fields on infinitely many sheets of Minkowski space; see Schroer, B., Swieca, J. A.: Phys. Rev. D 10, 480 (1974) and Ref. [9]

5. Rühl,W., Yunn,B.C.: Commun. math. Phys. 48, 215 (1976)

6. Rühl,W., Yunn,B.C.: Representations of the universal covering group of the conformal group belonging to the continuous principal series, Universität Kaiserslautern, Technical Report, October 1976

7. Rühl,W., Yunn,B.C.: Quantum fields with a multiplicative structure, Universität Kaiserslautern, Preprint, November 1976; beyond the ideas presented here, it contains details and gives results on the application of harmonic analysis

8. Rühl,W., Yunn, B.C.: Fortschr. Phys. 25, 83 (1977)

Communicated by R. Haag

Received February 2, 1977

1 In the terminology of Ref. [5] $s_{i j l}$ is a covariant kernel of the second kind. These are known only for two-dimensional Minkowski space. Though their existence for higher dimensional spaces is likely, there may be a finite degeneracy 
